\title{
Ética en la investigación científica
}

\section{Ethics in scientific research}

\section{Lisbeth Katerine Inguillay Gagñay}

Estudiante, Escuela Superior Politécnica de Chimborazo, Riobamba, Ecuador, lisbeth.inguillay@espoch.edu.ec, https://orcid.org/oooo-0002-9485-1206

\section{Silvia Lorena Tercero Chicaiza}

Estudiante, Escuela Superior Politécnica de Chimborazo, Riobamba, Ecuador, silviatercero72@gmail.com, https://orcid.org/oooo-0003-2462-1998

José López Aguirre

Magister en Administración y Dirección de Empresas, Escuela Superior Politécnica de Chimborazo, Riobamba, Ecuador, jfo731@gmail.com, https://orcid.org/oooo-00o19706-5115

\section{Resumen}

En el presente artículo se da a conocer sobre la ética en la investigación científica ya que es indispensable mantener una conducta ética en todos los trabajos de investigación, ensayos, tesis, etc. La ética es el comportamiento o conducta humana, es aquí en donde se diferencia lo bueno de lo malo, lo correcto de lo incorrecto, esta doctrina es la que le caracteriza al ser humano y nos da a conocer de los principios éticos que les caracterizan. No sólo se trata de cumplir con las obligaciones morales de los seres humanos hacia las personas que los rodean, sino que además deben mostrar su conducta ética en todos los aspectos de la

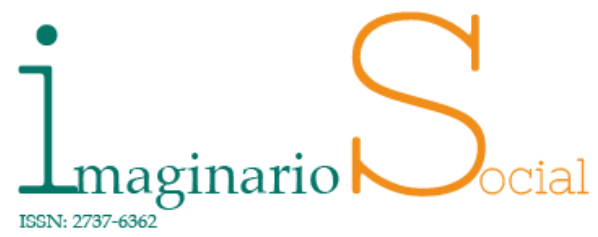

Imaginario Social e-ISSN: 2737-6362 enero - junio 2020 Vol. 3-1-2020 http://revista-

imaginariosocial.com/index.php/es/in dex

Recepción: 23 de agosto 2019 Aceptación: 11 de octubre 2019 $42-51$

Atribución/Reconocimiento-NoCo mercial- Compartirlgual 4.0 Licencia Pública Internacional - CC BY-NC-SA 4.0 https://creativecommons.org/licenses /by-nc- sa/4.0/legalcode.es 
vida cotidiana ya pueden ser en talleres, convenios, trabajos de investigación, tesis, ensayos, etc., es aquí en donde se debe aplicar la ética ya que así demuestran que sus trabajos son libres de plagio y además son frases o ideas propias que tienen la libertad de ser publicadas en cualquier medio. Todas personas que se dediquen a realizar trabajos de investigación deben tomar en cuenta acerca de la importancia de la ética en este tipo de trabajos, conocer acerca de las normas de citación y referenciación es importante ya que así los investigadores no corren el riesgo de ser denunciados o de robar ideas, frases o incluso artículos completos.

Palabras clave: ética, investigación, información, investigadores científicos, método científico.

\begin{abstract}
In this article, we will discuss ethics in scientific research, since it is essential to maintain ethical conduct in all research work, trials, theses, etc. Ethics is human behavior or conduct, it is here where good is distinguished from bad, right from wrong, this doctrine is what characterizes human beings and makes us aware of the ethical principles that characterize them. It is not only a matter of fulfilling the moral obligations of human beings towards the people around them, but they must also show their ethical conduct in all aspects of daily life, whether in workshops, conventions, research papers, theses, essays, etc., it is here that ethics must be applied, as they show that their work is free of plagiarism and are also their own phrases or ideas that are free to be published in any medium. Everyone who undertakes research work must be aware of the importance of ethics in this type of work. Knowledge of the rules of citation and referencing is important because researchers do not run the risk of being denounced or of stealing ideas, phrases or even whole articles.
\end{abstract}

Key word: ethics, research, information, scientific researchers, scientific method.

\title{
Introducción
}

Desde que los hombres viven en comunidad, la regulación moral de las acciones ha sido necesaria para el bienestar colectivo. Aunque los distintos sistemas morales se establecían 
sobre pautas arbitrarias, éstos evolucionaron a veces de forma absurda, a partir de la violación de los tabúes religiosos o de prácticas que primero fueron hábitos y luego costumbres, o asimismo de leyes impuestas por líderes para prevenir desequilibrios en el seno de la tribu, a medida que el tiempo pasa, los verdaderos investigadores se dan cuenta que realizar trabajos éticos es fundamental ya que así se hacen reconocidos y admirados por la sociedad, gracias a los avances científicos y tecnológicos los investigadores pueden recabar información relevante y eso es de gran ayuda para realizar sus trabajos de investigación. La ética debe ser aplicada en todas las etapas de la investigación, desde la planificación y la realización hasta la evaluación del proyecto de investigación. La ética de la investigación es completamente diferente en los tipos de comunidades educativas. Cada comunidad tiene su propio conjunto de valores morales. En Antropología la ética de la investigación fue formada para proteger a aquellos que están siendo investigados y para proteger al investigador de temas o sucesos que pueden ser peligrosos o que puedan hacer que cualquiera de las partes se sienta incómoda. Es una guía ampliamente observada que los antropólogos usan, especialmente cuando se hace trabajo de campo etnográfico (Morales, 2017).

Investigación Científica, es un proceso que, mediante la aplicación del método científico de investigación, se pretende obtener información relevante y concisa que ayuda a comprobar, verificar, complementar y aplicar en el conocimiento de las personas ya que es necesario aplicar algún tipo de investigación, la cual está muy ligada a los seres humanos, ésta posee una serie de pasos para lograr el objetivo planteado o para encontrar la información que se necesita, tiene como punto de partida el método científico y este es el tipo de estudio sistemático de la naturaleza que abarca diferentes técnicas de observación, normas de citación y referenciación, frases o ideas sobre el trabajo planificado y los métodos de comunicar los resultados experimentales y teóricos (Ecured, 2002).

\section{Materiales y Métodos}

La metodología empleada en el presente trabajo es exploratoria teniendo en cuenta que este tipo de investigación está orientada a descubrir o ampliar la información sobre un cierto tema desconocido que a través de varios medios se pretende recabar información acerca de dicha temática a fin de obtener nuevos datos, que ayuden a obtener nuevos conocimientos, hace referencia al primer acercamiento a un tema específico antes de abordarlo en un trabajo investigativo más profundo en un proceso 
para tener información básica relacionada con el problema de investigación. (Valducea, 2019).

Nos basamos en la recopilación de datos publicados en revistas científicas con el fin de aproximarnos al tema de investigación con una idea, los cuales fueron de gran ayuda para que nos familiaricemos con el mismo y esto nos permitió un mejor entendimiento de los significados y experiencias en el tema en cuestión, fundamentando de la información, a partir de este tipo de investigación se da a conocer de las verdaderas variables que afectan a la ética en las investigaciones científicas, así como cuales son los aspectos más interesantes que es pertinente abordar en la investigación.

\section{Resultados}

Desde que los hombres viven en comunidad, la regulación moral de las acciones ha sido necesaria para el bienestar colectivo. Aunque los distintos sistemas morales se establecían sobre pautas arbitrarias, éstos evolucionaron a veces de forma absurda, a partir de la violación de los tabúes religiosos o de prácticas que primero fueron hábitos y luego costumbres, o asimismo de leyes impuestas por líderes para prevenir desequilibrios en el seno de la tribu, por ende los filósofos griegos a partir del sexto siglo, teorizaron mucho sobre la acción moral, lo que llevó al posterior desarrollo de la ética (Ojeda, Quintero, Machado, 2007).

El alcance de la ética de la investigación científica está considerablemente respaldado desde muchos años atrás. Debido que, desde la aparición de dichos experimentos con los seres humanos, en el transcurso de la Segunda Guerra Mundial, en los que estuvieron involucrados no solo pocos médicos y científicos, sino que intervenía la humanidad, o al menos una parte de ella, es ahí cuando comprendieron la necesidad de monitorear la ética dentro de la Investigación Científica (Castillo Salazar, 2018).

Realmente es necesario que la ética esté inmersa con el desarrollo humano y de la misma manera con el progreso de la investigación científica. Cuando se concibe una investigación, la misma debe responder a una necesidad ante la cual se buscará la verdad a través del conocimiento, pero es imprescindible que todo ello tenga coherencia ética que garantice los hechos, los mismos que serán para el bien del hombre o la sociedad.

\section{Definiciones}

Según el autor (Barroso, 2020) "Ética es la ciencia filosófico-normativa y teórico-práctica que estudia los aspectos individuales y sociales de las personas en consecuencia del acto moral de 
los seres humanos, de manera que intervenga el conocimiento humano y la honestidad de cada uno con el propósito de llegar al bienestar común”. Ya que, en las investigaciones, la ética es algo más que asegurar el respeto a la persona y una consideración reflejada en el acápite de diseño metodológico, tratado muchas veces superficialmente.

Según el autor (Egg, 1992) la investigación es "un procedimiento reflexivo, sistemático, controlado y crítico que tiene por finalidad descubrir o interpretar los hechos y fenómenos, relaciones y leyes de un determinado ámbito de la realidad [...] una búsqueda de hechos, un camino para conocer la realidad, un procedimiento para conocer verdades parciales o, mejor, para descubrir no falsedades parciales”.

Consideramos que la investigación es el proceso más sensato, metódico y agotador que debemos llevar a cabo al momento de realizar el análisis del método científico, ya que comprende la distribución metodológica de la investigación que determina de manera general los puntos finales de cada procedimiento que se realiza para llegar a los resultados o conclusiones. Mientras que es posible emplear el espíritu científico sin investigación, sería imposible emprender una investigación a fondo sin emplear espíritu y método científico (Escalante, Comex).

\section{Ética en la Investigación Científica}

La ética de la investigación científica, es una descendencia de la ética personal, que está inmersa sobre cómo reaccionan los principios semejantes de la conducta moral del ser humano y se muestran específicamente en el contexto que fluye la vida del hombre y en este caso, el de la investigación científica y el trabajo académico, esto va avanzado de manera que surge la "mala conducta científica" o "mala conducta en la investigación". Esto quiere decir que la función principal dentro de la ética de la investigación científica es definir y delimitar con exactitud cuando no encontramos ante una mala conducta que pueda perjudicar los resultados finales de la investigación (Miranda, 2013).

La ética es una ciencia que tiene por objeto de estudio a la moral y la conducta humana, estos parámetros nos permiten estar más cerca del conocimiento que nos deja identificar lo que está bien y lo que está mal, teniendo en cuenta, la corrupción, la justicia y como actúa independientemente cada persona ya que esto es lo que guía nuestro comportamiento en diversidad de situaciones. En el diario vivir se forma una situación de la acción moral la cual, busca las razones que justifican la utilización de un sistema de referencia moral u otro (Guzmán, 2013).

\section{Aspectos éticos de la Investigación Científica}


Uno de los aspectos más importantes que encontramos para los requerimientos éticos del investigador científico es el predominio y el reconocimiento de los trabajos empleados para obtener información, así como la cualidad que describe a cada persona que haya intervenido en la realización del trabajo. Al momento de investigar es necesario no copiar ideas o usar fórmulas que no sean propias de la investigación constituye una práctica ajena a la ética e incluso comporta un robo intelectual o plagio científico (Upel, 2016).

Cuando se realiza una investigación se debe responder a una necesidad ante la que se buscará la verdad a través del conocimiento que van desarrollando durante el proceso de investigación. Es importante tener en cuenta que un trabajo de ética de las investigaciones debe incluir miembros con la calificación y la experiencia necesarias para revisar y evaluar los aspectos éticos, científicos y metodológicos de los proyectos que se sometan a su consideración (Rodríguez, 2018).

Se deben tomar en cuenta los aspectos que sencillamente son relevantes para cada estudiante o científico que va a realizar una investigación ya que en ese momento se presenta ante el gran desafío de plantearse un problema, enfrentarlo y orientarlo para que funcione de manera adecuada y se pueda llegar al resultado esperado y para ello juega un papel muy importante la responsabilidad moral y la ética personal como profesional.

\section{Importancia de la ética en la investigación}

En el mundo actual, el verdadero ejercicio de la libertad y la soberanía está en el conocimiento, se necesita la ciencia para disminuir los límites de la ignorancia y aumentar la capacidad para resolver los problemas. Un mejor estándar de vida puede lograrse en un país que disponga de recursos humanos altamente adiestrados formados en centros capaces de crear conocimientos $\mathrm{y}$ de formar profesionales imaginativos que puedan innovar y crear (Maracaibo, 2010).

Hay que comenzar por recordar que la historia de la investigación infortunadamente ha estado matizada por sucesos que opacan los maravillosos hallazgos científicos. Situación generada en gran medida por investigaciones adelantadas a ultranza y con el desconocimiento de los mínimos derechos de cada persona que intervinieron en ellas como sujetos de estudio; en muchas ocasiones sin que estos lo supieran (Pereira, 2012).

La importancia es que hay varios motivos por los cuales hay que someterse acotar a las normas que estén establecidas durante la investigación, ya que la validez del trabajo tendrá que ser verificados por la percepción del público para juzgar y aceptar nuevos resultados que se han publicado. Es necesariamente prescindible hacer una separación entendida para la conducta aceptable e inaceptable, esencialmente si los objetos de estudio son de humanos o animales, 
de la misma manera, algunos miembros de la comunidad de investigación continúan con la práctica de no informar apropiadamente los datos y los resultados científicos obtenidos.

\section{Principios éticos en la investigación}

La investigación aporta relevantes y esenciales hallazgos científicos innovadores que provocan el avance y el desarrollo de la humanidad, por ello deben existir principios que están recogidos en muchos campos o cuerpos normativos los cuales beneficien a los participantes en una investigación, de manera que maximicen los beneficios y minimicen los daños en el cual haya igualdad y garantía para la sociedad (Pérez, 2002).

Estos principios también se traducen en valores que son exigibles para que el trabajo de investigación pasa por la recta observación de las normas éticas.

Justicia: Consiste en dar a cada persona por igual lo que le corresponde

Tolerancia: Implica respetar las ideas y convicciones de los demás y permitir que se desarrollen.

Solidaridad: Fuerte vinculación con otras personas.

Libertad: Derecho natural de toda persona, sin ninguna distinción ya que es un derecho universal.

Responsabilidad: Responder por las consecuencias negativas y positivas de una acción realizada.

\section{Discusión}

El investigador debe utilizar los medios adecuados para la investigación es decir que deben respetar los derechos y los principales valores de la ética de la investigación los cuales permiten enseñar que los resultados de las investigaciones no se deben modificar, (...) y si estos se van a publicar, con mucha más razón. Ser honestos, llegar al fondo de los resultados y dar a conocerlos sin modificarlos, ya que esto implica la posibilidad de perder credibilidad de sus resultados. Se debe enseñar a ser comprometido con el trabajo, mostrarlo y darlo a conocer sin ajustes. Se busca la originalidad (Hirsch, 2010).

De acuerdo a los resultados obtenidos en los diferentes artículos científicos y páginas web, se presenció que es muy importante la ética en la investigación científica ya que les permite desarrollar de mejor manera la investigación siendo la ética uno de los actos reflexivos y entes que regulan la conciencia del investigador que busca conocimientos y soluciones a problemas 
no resueltos por medio del método científico el cual indica la ruta se va llevar a cabo durante el proceso de investigación y las técnicas que serán utilizadas. La ética se refiere a la ciencia de desarrollar teorías y reglas con respecto a las acciones humanas, partiendo de la filosofía que trata de la moral y las necesidades de la sociedad.

\section{Conclusiones}

La ética es un tema muy discutido en la actualidad, con muchas consideraciones distintas, que depende totalmente del ambiente cultural de cada sociedad, además se ha concluido que el uso adecuado de información forma a grandes investigadores ya que así realizan sus propios trabajos investigativos, usan sus propios conocimientos y eso hace que a las personas que los rodean les llame la atención y les incentiven a investigar.

La aplicación de la ética en trabajos de investigación es de suma importancia ya que así se respeta y se aplican correctamente los estilos normativos de citación y referenciación, además así se va dejando de lado el robo de ideas o de trabajos de otros autores que varias personas se apropian sin su consentimiento.

En las investigaciones se debe buscar la verdad, es decir evitar la falsificación de información, cambiar de correcto a lo incorrecto, uso de datos falsos, plagio o la manipulación de resultados a favor personal o de terceras personas. Estas prácticas conllevan a un problema para la comunidad científica, con efectos de dañar a la ciencia y a científicos.

Es importante que los centros educativos contribuyan, a través del desarrollo de investigaciones sobre aspectos éticos y morales, a promover y a establecer en el contexto educativo la cultura de la comunidad justa y, a marcar pautas en la búsqueda de una cultura moral altamente desarrollada para así evitar plagio de información.

\section{Referencias}

Acevedo, I. (2002). Aspectos Éticos en la Investigación Científica. Scielo, 1.

Barroso. (27 de mayo de 2020). Formación sociocultural. Obtenido de https://www.periodicodigitalgratis.com/13775/5-definiciones-de-etica-y-moral-dediferentes-autores-con131873

Castillo Salazar, D. (2018). Ética en la investigación científica y su inclusión en las ciencias de la salud. Informe, 2. 
Conicyt. (14 de junio de 2017). Obtenido de Conicyt: https://www.uss.cl/blog/aspectos-eticosla-investigacion/

Ecured. (2 de 7 de 2002). Obtenido de Ecured: https://www.ecured.cu/Investigaci\%C3\%B3n_cient\%C3\%ADfica

Egg, A. (1992). Universidad Santo Tomas. Redalyc.

Escalante, C. (8 de 4 de 2015). Comex. Obtenido de Comex: http://ual.dyndns.org/biblioteca/metodologia_investigacion/pdf/unidad_o2.pdf

Escalante, C. (s.f.). Comex. Recuperado el 8 de 6 de 2020, de Comex: http://ual.dyndns.org/biblioteca/metodologia_investigacion/pdf/unidad_o2.pdf

Galán Amador, M. (2019). Ética de la Investigación. Iberoamérica, 2.

Gómez, P. (2012). Principios de la Ética en la Investigación. Redalyc, 4.

Guzmán, J. (2013). La ética en la Investigación Científica. Periodismo Critico, 2.

Hirsch, A. (7 de 9 de 2010). Redytus. Obtenido de Redytus: file://C:/Users/REDYTUS/Downloads/DialnetPrincipalesValoresDeLaEticaDeLaInvestigacionQueSeP-3619789.pdf

Mantilla, J. (2011). Ética e Investigación. Scielo, 6.

Maracaibo. (2010). Importancia de la Investigación. Scielo, 2.

Miranda, A. (2 de 8 de 2013). Scielo. Obtenido de Scielo: https://scielo.conicyt.cl/scielo.php?pid=So71834372013000200016\&script=sci_arttext\&tlng=es.

Montecinos, A. (2013). Ética de la Investigación Científica. Scielo, 2.

Morales, J. (2017). Ética en la Investigación Científica. Scielo, 3.

Ojeda, Quintero, Machado, J. (2007). La ética en la investigación. Redalyc, 3.

Pereira. (2012). Importancia de la Ética en la Investigación Científica. Scielo, 1.

Pérez. (2002). Importancia de ética en la investigación. Scielo, 4.

Rodríguez, N. (8 de 4 de 2018). Informe. Obtenido de Informe: http://www.revactamedicacentro.sld.cu/index.php/amc/article/view/88o/1157\#: :tex 
$\mathrm{t}=$ En\%2olas\%2oinvestigaciones\%20cient\%C3\%ADficas\%2olos,por\%20ende\%2C\%20e 1\%20desarrollo\%2ocient\%C3\%ADfico.

Upel. (29 de 3 de 2016). Obtenido de Upel: https://eticainvestigativa.wordpress.com/2016/o3/29/aspectos-eticos-en-lainvestigacion-cientifica/

Valducea, M. (2019). Metodología de la investigación. Obtenido de https://selinea.unidep.edu.mx/files/528to832_r649220160427120000289061.pdf 